Ann. Biol. anim. Bioch. Biophys., 1974, 14 (3), 575-578.

NOTE

\title{
INDIVIDUALITÉ MUSCULAIRE CHEZ LE BOVIN : ÉTUDE DE LA CONCENTRATION EN DNA DE QUELQUES MUSCLES
}

\author{
M. ANSAY \\ avec la collaboration technique de J. Roupain \\ Chaire de Génétique, Faculté de Médecine vétérinaire, \\ 45, Rue des Vétérinaires, \\ 1070 Bruxelles (Belgique)
}

RÉSUMÉ

La détermination de la concentration en DNA de divers muscles du veau mâle de 83 kilos a permis d'établir d'importantes différences. Présentés par ordre d'importance décroissante de la teneur en DNA, les 5 groupes suivants ont été délimités : I) le cœur ; 2) le masséter et le diaphragme ; 3) les muscles du cou; 4) les muscles des extrémités digitées ; 5) les muscles du rachis et des parties proximales des membres.

Chez les animaux domestiques, l'étude comparée des muscles révèle d'importantes différences qui se manifestent à divers niveaux :

$I^{\circ}$ au niveau de la composition (teneur en collagène, en élastine, équipement enzymatique) (Boccard, I968; BeNDAll, I967; ANSAy, I974);

$2^{\circ}$ au niveau de la réponse apportée à des stimuli divers tels que 1'hypertrophie musculaire, la croissance, la dégénérescence musculaire (DUMONT et BOCCARD, 1972 ; HANSET et ANSAY, I972; Butterfield et Berg, I966; Briskey, 1964 ; Ouhayoun, I973).

L'objet de ce travail est de comparer divers muscles bovins du point de vue de leur concentration respective en DNA-P.

Le matériel animal est constitué par 5 veaux de sexe mâle, abattus au poids constant de 83 kilos et d'un âge moyen de 65 jours. Ils sont issus de cinq vaches de race Pie-noive (Hollandaise) et d'un seul taureau de race de Moyenne et Haute Belgique. 
Préalablement à l'extraction (deux fois 20 minutes en acide perchlorique normal à $70^{\circ} \mathrm{C}$ ), la méthode générale de Munro et Fleck (I966) a été suivie (Ansay et Grilet, r973). Le déoxyribose a été déterminé par la méthode de BuRTon (I956).

L'analyse de variance, à deux voies, (" entre muscles " et " entre individus ") a montré que les différences entre muscles étaient très hautement significatives (tabl. I).

TABLEAU I

Analyse de variance

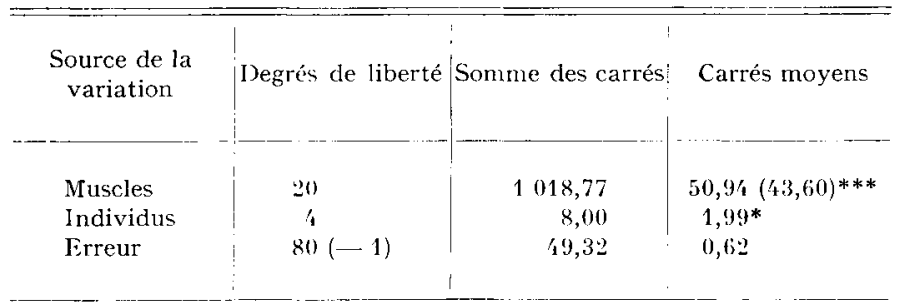

N.B. : Analyse effectuée selon SNEdicor et Cochran (1967), pour une donnée manquante (pour le coeur).

*** Test de $\mathrm{F}$ significatif au seuil 1 p. 1000.

* Test de $\mathrm{F}$ significatif au seuil 5 p. 100.

L'examen du tableau 2, qui rapporte les concentrations moyennes propres à chaque muscle et les plus petites différences significatives à divers seuils de signification permet de distinguer cinq types de muscles :

I) le cœur avec une concentration moyenne de $18,2 \mathrm{I}$ ( $\mathrm{mg} / \mathrm{roo} \mathrm{g}$ de poids frais) ;

2) le masseter, le diaphragme : i I,34 et 8,i 5 ;

3) une série de muscles du cou (splenius, semispinalis capitis, obliquus capitis caudalis, rhomboideus) : de 8,39 à 6,13 ;

4) quelques muscles des extrémités digitées avec des concentrations supérieures à 5 ;

5) un groupe de muscles situés soit à la partie proximale des membres soit à hauteur du rachis (dos et lombes) et caractérisés par des valeurs intermédiaires entre 5,13 (longissimus dorsi) ct 4,34 (semitendinosus).

Ces observations démontrent qu'en termes de "nucléarité " (nombre de noyaux/gramme de tissu frais, en admettant la constance de la concentration nucléaire moyenne en DNA), des différences importantes existent entre les muscles de la bête bovine.

Cependant, la part respective des noyaux appartenant à la fibre proprement dite et au compartiment intercellulaire n'est pas précisée. Il n'est pas non plus possible de tirer de ces résultats des renseignements sur la grandeur de la fibre musculaire elle-même. De tels renseignements ressortent surtout de l'approche histologique (Sснміт et Dumont, 1969, 1972).

On peut cependant suggérer en se fondant sur les observations d'un mémoire précédent (ANSAY, 1973) que les muscles avec la teneur en DNA la plus grande sont aussi ceux dont le métabolisme est du type le plus oxydatif. Ce sont précisément les muscles les moins hypertrophiés chez l'animal culard.

L'étude du comportement biochimique et histochimique de ces deux types de muscles (type splenius et type biceps femoris) chez des animaux culards et normaux de même âge devrait apporter d'utiles renseignements sur le déterminisme de l'hypermuscularité du culard. Le phéno- 
TABLEAU 2

Concentration en $D N A-P$ (mg/Ioo $\mathrm{g}$ de poids frais) de divers muscles

\begin{tabular}{|c|c|c|}
\hline Coeur . . & 18,21 & Glutaeus medius . . \\
\hline Masseter ......... & 11,34 & 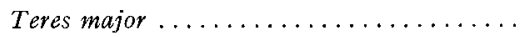 \\
\hline Splenius .......... & 8,39 & Flexor digitorum longus (Pedis) . ...... \\
\hline Diaphragme $\ldots \ldots \ldots \ldots \ldots \ldots \ldots$ & 8,15 & Pectinaeus $\ldots \ldots \ldots \ldots \ldots \ldots \ldots \ldots$ \\
\hline Semispinalis capitis ............... & 7,10 & 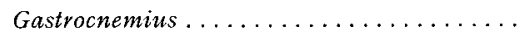 \\
\hline Obliquus capitis caudalis ............ & 6,61 & 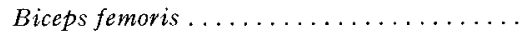 \\
\hline Rhomboideus ................. & 6,13 & Deltoideus $\ldots \ldots \ldots \ldots \ldots \ldots \ldots \ldots$ \\
\hline Flexor digitorum superficialis $\ldots . \ldots \ldots$ & 6,06 & Tensor fasciae latae ................. \\
\hline Flexor carpi radialis ...... & 5,25 & Rectus femoris . ................. \\
\hline Longissimus dorsi .............. & 5,13 & 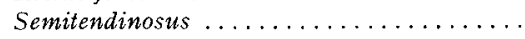 \\
\hline Psoas major $\ldots \ldots \ldots \ldots \ldots \ldots \ldots$ & 5,11 & \\
\hline
\end{tabular}

Plus petite différence signi ficative entre le coeur et tout autre muscle au seuil de 5 p. $100: 1,05 ; 1$ p. 100 : 1,$39 ; 1$ p. $1000: 1,80$.

Plus petite différence significative entre deux muscles quelconques au seuil de 5 p. $100: 0,99 ; 1$ p. 100 : 1,$31 ; 1$ p. $1000: 1,70$.

mène culard pourrait ne toucher qu'un seul type de fibre, que ce soient les fibres "blanches" (AsHMORE et Robinson, I969) ou les fibres aptes à subir la transformation " $\alpha$ Red $\rightarrow \alpha$ White" (Ashmore et DOERR, 197I), les gros muscles possédant ce type de fibre en proportion plus grande.

Reçu pour publication en décembre 1973.

\section{REMERCIEMENTS}

Ce travail a été exécuté sous les auspices de l'Institut pour l'encouragement de la Recherche Scientifique dans 1'Industrie et l'Agriculture (I. R. S. I. A.).

Nous tenons à remercier M. le Professeur HANSET pour ses conseils dans l'exécution des calculs statistiques.

\section{SUMMARY \\ MUSCULAR INDIVIDUALITY IN CATTLE : STUDY OF DNA CONTENT IN SOME MUSCLES}

Wide differences are shown in the DNA content of various muscles of the 83-kilo male calf. Presented in decreasing order of DNA content, the following 5 groups are defined : I) heart; 2) masseter and diaphram; 3) neck muscles ; 4) muscles of digital extremities; 5) muscles of rachis and proximal parts of limbs.

\section{RÉFÉRENCES BIBLIOGRAPHIQUES}

ANSAY M., I974. Individualité musculaire chez la bête bovine. I. Étude de l'équipement enzymatique de quelques muscles. Ann. Biol. anim. Bioch. Biophys., 14, 471-486.

Ansay M., Gillet A., I973. Étude biochimique comparée du tissu musculaire des bovidés culards et normaux, après la naissance. I. Les acides nucléiques et l'azote total. Ann. Méd. Vét., 117, 39I-400. 
Ashmore C. R., Robinson D. W., I969. Hereditary muscular hypertrophy in the bovine. I. Histological and biochemical characterizations. Proc. Soc. Exptl. Biol. Méd, 132, 548-554.

Ashmore C. R., DoERR L., I97I. Postnatal development of fiber types in normal and dystrophic skeletal muscle of the chick. Exp. Neurol., 30, 43I-446.

Bendall J. R., 1967. The elastin content of various muscles of beef animals. J. Sci. Fd Agric., 18, $553-55^{8}$.

Boccard R., r968. Variation de la teneur en hydroxyproline de muscles de porcs Large White et Piétrain. Ann. Zootech., 17, 7I-75.

Briskey E. J., I964. Etiological status and associated studies of pale, soft, exsudative porcine musculature. Adv. Food Res., 13, 89-1 78 .

BURToN K., 1956. A study of the conditions and mechanisms of the diphenylamine reaction for the colorimetric estimation of deoxyribonucleic acid. Biochem. $J, 62,315-322$.

Butterfield R. M., Berg R. T., I966. A classification of bovine muscles based on their relative growth patterns. Res. Vet. Sci., 7, 326-332.

Dumont B.-L., Boccard R., I972. Conséquences de l'hypertrophie musculaire héréditaire des bovins sur la musculature. II. Importance relative des différents muscles. Ann. Génét. Sél. anim., 4, I30-I3I.

Hanset R., ANSAy M., 1972. Régions privilégiées d'hypertrophie musculaire, chez le bovin culard. Ann. Méd. Vêt., 116, I7-25.

MunRo H. N., Fleck A., I966. The determination of nucleic acids, in Methods of Biochemical analysis Éd. D. Glick, Interscience Publishers 14, II3-I76.

Ouhayoun J., 1973. Relations entre le métabolisme du tissu musculaire et la qualité de la viande. (Rencontre I. N. R. A.-A. B. R. O., Paris, septembre 1973).

Schmitт O., Dumont B.-L., r969. Méthodes d'analyses de la structure musculaire. Ann. Biol. anim. Bioch. Biophys., 9, 123-134.

Schmitr O., Dumont B. L., I972. Conjonctif et croissance musculaire. Ann. Biol. anim. Bioch. Biophys. 12, 667-672.

SNedecor G. W., Cochran W. G., 1967. Statistical methods. Sixth edition. Iowa State University. 